\title{
Universal Gröbner Bases of Colored Partition Identities
}

\author{
Tristram Bogart, Ray Hemmecke, and Sonja Petrović
}

CONTENTS

1. Introduction

2. Partitions, Scrolls, and the Main Theorem

3. Geometry of Universal Gröbner Bases and Graver Bases

4. Computations

5. Graver Complexity and the Remainder of the Proof References

2000 AMS Subject Classification: 05E40, 13P10, 11P84, 90C27

Keywords: Graver bases, Universal Gröbner bases, partition identities, colored partitions, rational normal scrolls, state polytope, toric ideal
Associated to any toric ideal are two special generating sets: the universal Gröbner basis and the Graver basis, which encode polyhedral and combinatorial properties of the ideal, or equivalently, its defining matrix. If the two sets coincide, then the complexity of the Graver bases of the higher Lawrence liftings of the toric matrices is bounded.

While a general classification of all matrices for which both sets agree is far from known, we identify all such matrices within two families of nonunimodular matrices, namely, those defining rational normal scrolls and those encoding homogeneous primitive colored partition identities. This also allows us to show that higher Lawrence liftings of matrices with fixed Gröbner and Graver complexities do not preserve equality of the two bases.

The proof of our classification combines computations with the theoretical tool of Graver complexity of a pair of matrices.

\section{INTRODUCTION}

Toric ideals, their generating sets, and Gröbner bases play a prominent role in many different areas, such as algebraic geometry [Fulton 93], commutative algebra [Villarreal 01], graph theory, integer programming [Sturmfels 96], and algebraic statistics [Drton et al. 09]. The underlying combinatorics allow for relatively efficient computation and have inspired several important algorithms, for example those implemented in software packages 4 ti 2 and Gfan. ${ }^{1}$ In this note, we explore the problem of equality of two special generating sets of toric ideals for two families of projective varieties.

For a matrix $A \in \mathbb{Z}^{d \times n}, \mathrm{I}_{\mathrm{A}}$ will denote its associated toric ideal: $\mathrm{I}_{\mathrm{A}}=\left\langle\mathbf{x}^{\mathbf{u}^{+}}-\mathbf{x}^{\mathbf{u}^{-}}: A \mathbf{u}=\mathbf{0}\right\rangle$, where we write $\mathbf{u}=\mathbf{u}^{+}-\mathbf{u}^{-}$such that both $\mathbf{u}^{+}$and $\mathbf{u}^{-}$have only nonnegative coordinates and disjoint support. The minimal universal Gröbner basis of $\mathrm{I}_{\mathrm{A}}$, denoted by $\mathcal{U}(A)$, is the

\footnotetext{
${ }^{1}$ Available at www.4ti2.de and http://www.math.tu-berlin.de/ $\sim$ jensen/software/gfan/gfan.html respectively.
} 
union of all of the (finitely many) reduced Gröbner bases of $\mathrm{I}_{\mathrm{A}}$. It is contained in the Graver basis $\mathcal{G}(A)$, consisting of all primitive binomials in the ideal, where a binomial $\mathbf{x}^{\mathbf{u}^{+}}-\mathbf{x}^{\mathbf{u}^{-}} \in \mathrm{I}_{\mathrm{A}}$ is primitive if there is no other binomial $\mathbf{x}^{\mathbf{v}^{+}}-\mathbf{x}^{\mathbf{v}^{-}} \in \mathrm{I}_{\mathrm{A}}$ such that $\mathbf{x}^{\mathbf{v}^{+}}$divides $\mathbf{x}^{\mathbf{u}^{+}}$and $\mathbf{x}^{\mathbf{v}^{-}}$divides $\mathbf{x}^{\mathbf{u}^{-}}$.

It is known that the universal Gröbner and Graver bases agree for some special toric ideals (e.g., toric ideals of unimodular matrices [Sturmfels 92, Sturmfels and Thomas 97]). However, a general classification of matrices for which $\mathcal{U}(\mathcal{A})=\mathcal{G}(\mathcal{A})$ is not known. The equality of the two sets has an important consequence for higher Lawrence liftings of $A$, as we discuss below.

In our main result, Theorem 2.2, we solve this classification problem for two families of nonunimodular matrices indexed by partitions. A motivation for this work is provided by the connection between partition identities and Graver bases of rational normal scrolls, explored in [Petrović 08]. Rational normal scrolls are a classical family of projective varieties representing one of the partition-indexed families we study. The work [Eisenbud and Harris 87] surveys the geometry of these scrolls: they belong to the family of nondegenerate projective varieties of minimum possible degree, just one more than the codimension. The case $c=1$ represents the rational normal curve in $\mathbb{P}^{n-1}$. They are studied in [Conca et al. 07] from the perspective of Gröbner theory: in particular, all of their Cohen-Macaulay initial ideals are classified. A classification of the remaining initial ideals, or equivalently, a determination of the remainder of the Gröbner fan, remains open. This question is related to universal Gröbner bases, and one approach to this problem is to first understand an approximation of the set $\mathcal{U}(\mathcal{A})$.

In proving our result, we study the complexity of the elements in the two bases. For matrix families known as $N$-fold matrices of a pair $(A, B)$, this complexity is bounded as $N$ grows. These bounds are called the Graver complexity $g(A, B)$, defined in [Santos and Sturmfels 03], and the Gröbner complexity $u(A, B)$, defined in [Hemmecke and Nairn 09]. $N$-fold matrices are also known as generalized higher Lawrence liftings of a matrix $A$ with respect to the matrix $B$ (e.g., [Santos and Sturmfels 03]).

Equality of the Graver and universal Gröbner bases has an important consequence for higher Lawrence liftings of $A$ : if $\mathcal{G}(A)=\mathcal{U}(A)$, then $u(A, B)=g(A, B)$ for all integer matrices $B$ of suitable dimension (see [Hemmecke and Nairn 09, Theorem 1]). However, the question whether $N$-fold matrices with fixed Gröbner and Graver complexities preserve equality of universal Gröbner and Graver bases was left open. Our two families of matrices demonstrate with infinitely many examples that the answer to this question is negative (see Corollary 5.5).

More generally, if the two sets do not coincide, it is not clear whether the ratio $|\mathcal{U}(A)| /|\mathcal{G}(A)|$ tends to 0 as the size of the matrix $A$ increases within a family. It is also an interesting open question whether the special structure of our matrices implies that there are only finitely many fundamental counterexamples to equality of universal Gröbner basis and Graver basis, from which all other counterexamples can be derived. Our results and methods offer a first step toward understanding such questions, which still pose a computational challenge.

\section{PARTITIONS, SCROLLS, AND THE MAIN THEOREM}

Let us introduce the two families of matrices we will study.

Definition 2.1. Given a partition $n_{1} \geq n_{2} \geq \cdots \geq n_{c}$ of a positive integer $n$, define

$$
\begin{aligned}
& A_{S\left(n_{1}-1, \ldots, n_{c}-1\right)} \\
& \quad:=\left[\begin{array}{ccccccccccc}
1 & 2 & \cdots & n_{1} & 1 & \cdots & n_{2} & \cdots & 1 & \cdots & n_{c} \\
1 & 1 & \cdots & 1 & 0 & \cdots & 0 & \cdots & 0 & \cdots & 0 \\
0 & 0 & \cdots & 0 & 1 & \cdots & 1 & \cdots & 0 & \cdots & 0 \\
\vdots & \vdots & & \vdots & & & \ddots & & \\
0 & 0 & \cdots & 0 & 0 & \cdots & 0 & \cdots & 1 & \cdots & 1
\end{array}\right]
\end{aligned}
$$

and

$$
\begin{aligned}
& A_{H\left(n_{1}, \ldots, n_{c}\right)} \\
& \quad:=\left[\begin{array}{ccccccccccc}
1 & 1 & \cdots & 1 & 1 & \cdots & 1 & \cdots & 1 & \cdots & 1 \\
1 & 2 & \cdots & n_{1} & 0 & \cdots & 0 & \cdots & 0 & \cdots & 0 \\
0 & 0 & \cdots & 0 & 1 & \cdots & n_{2} & \cdots & 0 & \cdots & 0 \\
\vdots & \vdots & & \vdots & & & \ddots & & \\
0 & 0 & \cdots & 0 & 0 & \cdots & 0 & \cdots & 1 & \cdots & n_{c}
\end{array}\right] .
\end{aligned}
$$

The toric ideal associated to the first matrix is the defining ideal of the $c$-dimensional rational normal scroll $S:=S\left(n_{1}-1, \ldots, n_{c}-1\right)$ in $\mathbb{P}^{n-1}$; see [Petrović 08 , Lemma 2.1]. This ideal is generated by the $2 \times 2 \mathrm{mi}-$ nors of the matrix $M=\left[M_{n_{1}}|\cdots| M_{n_{c}}\right]$ of indeterminates, where

$$
M_{n_{j}}=\left[\begin{array}{ccc}
x_{j, 1} & \cdots & x_{j, n_{j}-1} \\
x_{j, 2} & \cdots & x_{j, n_{j}}
\end{array}\right] .
$$

These two families of toric ideals have a nice combinatorial interpretation. The binomials $x_{a_{1}} x_{a_{2}} \cdots x_{a_{k}}$ 
$-x_{b_{1}} x_{b_{2}} \cdots x_{b_{k}}$ in the ideal $I_{S\left(n_{1}-1\right)}$ of a rational normal curve encode homogeneous partition identities:

$$
a_{1}+\cdots+a_{k}=b_{1}+\cdots+b_{k},
$$

where $a_{1}, \ldots, a_{k}, b_{1}, \ldots, b_{k}$ are positive integers, not necessarily distinct [Diaconis 96]. Similarly, the binomials in the ideal $I_{S}$ of a rational normal scroll encode color-homogeneous colored partition identities (colorhomogeneous cpi's) [Petrović 08]:

$$
\begin{aligned}
& a_{1,1}+\cdots+a_{1, k_{1}}+\cdots+a_{c, 1}+\cdots+a_{c, k_{c}} \\
& \quad=b_{1,1}+\cdots+b_{1, k_{1}}+\cdots+b_{c, 1}+\cdots+b_{c, k_{c}} .
\end{aligned}
$$

For example,

$$
1_{1}+5_{1}+1_{2}+5_{2}=2_{1}+6_{1}+2_{2}+2_{2}
$$

is a color-homogeneous cpi encoded by the binomial $x_{1,1} x_{1,5} x_{2,1} x_{2,5}-x_{1,2} x_{1,6} x_{2,2}^{2}$. (We have used black and dark gray to represent two colors, say red and blue.) Among such identities, those with no proper subidentities are again called primitive and are encoded by the elements of $\mathcal{G}\left(A_{S}\right)$ [Petrović 08].

Similarly,

$$
1_{1}+5_{1}+1_{2}=3_{1}+1_{2}+3_{2}
$$

is a cpi that is homogeneous, but not colorhomogeneous, and is encoded by the binomial $x_{1,1} x_{1,5} x_{2,1}-x_{1,3} x_{2,1} x_{2,3}$. Primitive homogeneous cpi's correspond to the elements of $\mathcal{G}\left(A_{H}\right)$.

This attractive characterization of the Graver bases makes it especially useful to classify which scrolls have the property that the universal Gröbner basis is equal to the Graver basis.

Within these two families of matrices, we will classify those for which the universal Gröbner and Graver bases coincide. To state the classification, we use the dominance partial order on partitions: $\left(n_{1}, \ldots, n_{c}\right) \preceq\left(m_{1}, \ldots, m_{d}\right)$ if $c \leq d$ and $n_{j} \leq m_{j}$ for $j=1, \ldots, c$. In this case, we say that $S\left(m_{1}, \ldots, m_{d}\right)$ dominates $S\left(n_{1}, \ldots, n_{c}\right)$, and $H\left(m_{1}, \ldots, m_{d}\right)$ dominates $H\left(n_{1}, \ldots, n_{c}\right)$.

Theorem 2.2. The universal Gröbner basis of $S=S\left(n_{1}-\right.$ $\left.1, \ldots, n_{c}-1\right)$ equals its Graver basis if and only if $S$ does not dominate $S(6), S(5,4)$, or $S(4,3,2)$.

The universal Gröbner basis of $H=H\left(n_{1}, \ldots, n_{c}\right)$ equals its Graver basis if and only if $H$ does not dominate $H(7), H(6,2)$, or $H(4,3)$.

The proof will proceed as follows. First, we review the geometry of Graver bases and universal Gröbner bases as discussed in [Sturmfels 96, Sturmfels and Thomas 97].
This geometry allows us to conclude that equality of the two sets is preserved under passing to a scroll lower in the dominance order. Next we use computational tools to exhibit explicit Graver elements demonstrating inequality in each dominance-minimal case mentioned in the theorem, and also to verify equality in certain other cases. The computational step is critical to our result in that it was very hard to find minimal counterexamples to equality by hand. But with hindsight, we were able to verify them by hand. We then apply Graver complexity to reduce the infinite list of remaining cases, that is, those for which we need to verify equality, to the finitely many cases we have considered.

\section{GEOMETRY OF UNIVERSAL GRÖBNER BASES AND GRAVER BASES}

Fix a graded toric ideal $\mathrm{I}_{\mathrm{A}}$ by fixing $A \in Z^{d \times n}$ such that the row span of $A$ contains a vector strictly in the positive orthant of $\mathbb{R}^{n}$. Then for any $\mathbf{b} \in \mathbb{N}^{d}$, the set $P_{\mathbf{b}}^{I}:=\operatorname{conv}\left(\left\{\mathbf{u}: A \mathbf{u}=\mathbf{b}, \mathbf{u} \in \mathbb{Z}_{+}^{n}\right\}\right)$ is a polytope, called the fiber of $\mathbf{b}$.

Proposition 3.1. [Sturmfels 96] An integer vector $\mathbf{u}^{+}-$ $\mathbf{u}^{-}$(with $\left.\mathbf{u}^{+}, \mathbf{u}^{-} \geq \mathbf{0}\right)$ lies in $\mathcal{U}(A)$ if and only if the line segment $\left[\mathbf{u}^{+}, \mathbf{u}^{-}\right]$is an edge of the fiber $P_{A \mathbf{u}^{+}}^{I}$ and contains no lattice points other than its endpoints.

Proposition 3.1 implies a well-known and very useful fact, namely, that certain projections preserve elements in the universal Gröbner and Graver bases:

Corollary 3.2. [Sturmfels 96, Proposition 4.13] Suppose $\mathbf{u} \in \operatorname{ker}_{\mathbb{Z}} A$ and $\sigma \subseteq[n]$ are such that $\mathbf{u}_{i}=0$ for $i \notin \sigma$. Let $A_{\sigma}$ be the submatrix of $A$ of columns indexed by $\sigma$, and let $\mathbf{u}_{\sigma}$ be the projection of $\mathbf{u}$ to $\mathbb{R}^{\sigma}$. Then:

(a) $\mathbf{u} \in \mathcal{U}(A)$ if and only if $\mathbf{u}_{\sigma} \in \mathcal{U}\left(A_{\sigma}\right)$.

(b) $\mathbf{u} \in \mathcal{G}(A)$ if and only if $\mathbf{u}_{\sigma} \in \mathcal{G}\left(A_{\sigma}\right)$.

Proof. To prove claim (a), observe that since the hyperplanes $x_{i}=0(i \notin \sigma)$ do not pass through the interior of $P_{A \mathbf{u}^{+}}$, the polytope $P_{A_{\sigma} \mathbf{u}_{\sigma}^{+}}$is a face of $P_{A \mathbf{u}^{+}}$. It follows that the segment $\left[\mathbf{u}^{+}, \mathbf{u}^{-}\right]$is an edge of $P_{A_{\sigma} \mathbf{u}_{\sigma}^{+}}$if and only if it is an edge of $P_{A \mathbf{u}^{+}}$.

To prove claim (b), simply observe that the minimality property of $\mathbf{x}^{\mathbf{v}^{+}}-\mathbf{x}^{\mathbf{v}^{-}} \in \mathrm{I}_{\mathrm{A}}$ is the same for both matrices $A$ and $A_{\sigma}$, since the variables indexed by elements of $\sigma$ do not appear in either case. 
Corollary 3.2 immediately implies that equality of the two sets is inherited by submatrices:

Corollary 3.3. Let $A_{\sigma}$ be obtained from $A$ by first choosing the submatrix of $A$ consisting of the columns indexed by $\sigma \subseteq[n]$ and then eliminating some or all rows consisting entirely of zeros. Then $\mathcal{U}(A)=\mathcal{G}(A)$ implies $\mathcal{U}\left(A_{\sigma}\right)=\mathcal{G}\left(A_{\sigma}\right)$.

By inspection of the matrices associated to a pair of partitions related in dominance order, we then obtain the desired consequence for our classification problem.

Corollary 3.4. Suppose $\left(n_{1}, \ldots, n_{c}\right) \prec\left(m_{1}, \ldots, m_{d}\right)$. Then $\mathcal{U}\left(A_{S\left(m_{1}, \ldots, m_{d}\right)}\right)=\mathcal{G}\left(A_{S\left(m_{1}, \ldots, m_{d}\right)}\right) \quad$ implies $\mathcal{U}\left(A_{S\left(n_{1}, \ldots, n_{c}\right)}\right)=\mathcal{G}\left(A_{S\left(n_{1}, \ldots, n_{c}\right)}\right)$.

Similarly, $\mathcal{U}\left(A_{H\left(m_{1}, \ldots, m_{d}\right)}\right)=\mathcal{G}\left(A_{H\left(m_{1}, \ldots, m_{d}\right)}\right) \quad$ implies $\mathcal{U}\left(A_{H\left(n_{1}, \ldots, n_{c}\right)}\right)=\mathcal{G}\left(A_{H\left(n_{1}, \ldots, n_{c}\right)}\right)$.

\section{COMPUTATIONS}

Proposition 3.1 allows us to check computationally whether $\mathcal{U}(A)=\mathcal{G}(A)$ for any given matrix $A$ as follows. First, we compute the Graver basis $\mathcal{G}(A)$; this can be done rapidly with the software package $4 \mathrm{ti} 2$. Then we test the statement in Proposition 3.1 for every $\mathbf{g} \in \mathcal{G}(A)$. To check this condition, we first enumerate the set $V$ of all lattice points in the polytope $P_{A \mathbf{g}^{+}}$and then check whether $\mathbf{g}$ is an edge of this polytope that contains no interior lattice point. This can be done by first testing whether $\mathbf{g}^{+}$and $\mathbf{g}^{-}$are vertices of $P_{A_{\mathbf{g}^{+}}}$(that is, they are not convex combinations of $V \backslash\left\{\mathbf{g}^{-}\right\}$and of $V \backslash\left\{\mathbf{g}^{+}\right\}$, respectively). If $\mathbf{g}^{+}$and $\mathbf{g}^{-}$are vertices of $P_{A \mathbf{g}^{+}}$, then $\mathbf{g}$ is an edge with no integer point in its interior if and only if there does not exist a decomposition

$$
\mathbf{g}=\mathbf{g}^{+}-\mathbf{g}^{-}=\sum_{\mathbf{u} \in V \backslash\left\{\mathbf{g}^{+}, \mathbf{g}^{-}\right\}} \lambda_{\mathbf{u}}\left(\mathbf{u}-\mathbf{g}^{-}\right)
$$

with nonnegative real coefficients $\lambda_{\mathbf{v}}$. This feasibility problem can be decided by any code that solves linear programs. We applied the commercial solver CPLEX Callable Library 9.1.3.

The problem of enumerating points in a lattice polytope of varying dimension is still computationally difficult. In practice, however, this is better than computing universal Gröbner bases. For small scrolls, the universal Gröbner basis can be computed by the software package $\mathrm{Gfan}$. Once we reached nine variables, this computation failed to terminate. Even when the universal Gröbner basis itself is not especially large, each of its binomials typ- ically occurs in many different reduced Gröbner bases, each of which Gfan must compute in order to verify that the entire universal Gröbner basis has been found.

Our computational method produced the dominanceminimal examples in the main theorem. In each of these cases, it is not difficult to verify inequality by hand, as we demonstrate below.

Lemma 4.1. The universal Gröbner basis and the Graver basis do not coincide for the defining matrices of $S(6)$, $S(5,4), S(4,3,2), H(7), H(6,2)$, and $H(4,3)$.

Proof. The defining matrix of $S(6)$ is

$$
A_{S(6)}=\left(\begin{array}{lllllll}
1 & 2 & 3 & 4 & 5 & 6 & 7 \\
1 & 1 & 1 & 1 & 1 & 1 & 1
\end{array}\right) .
$$

Consider the vector $\mathbf{g}=(1,-1,1,-1,-1,0,1) \in$ $\operatorname{ker}\left(A_{S(6)}\right)$ and the three vectors $\mathbf{u}_{1}=(1,0,0,0,2,0,0)$, $\mathbf{u}_{2}=(0,2,0,0,0,0,1), \mathbf{u}_{3}=(0,0,1,2,0,0,0)$ in the fiber $\left\{\mathbf{u}: A_{S(6)} \mathbf{u}=A_{S(6)} \mathbf{g}^{+}, \mathbf{u} \in \mathbb{Z}_{+}^{7}\right\}$ of $\mathbf{g}$. Notice that

$$
\begin{aligned}
\mathbf{g} & =\mathbf{g}^{+}-\mathbf{g}^{-} \\
& =1 \cdot\left(\mathbf{u}_{1}-\mathbf{g}^{-}\right)+1 \cdot\left(\mathbf{u}_{2}-\mathbf{g}^{-}\right)+1 \cdot\left(\mathbf{u}_{3}-\mathbf{g}^{-}\right),
\end{aligned}
$$

and thus $\mathbf{g}$ is not an edge of $P_{\mathbf{g}^{+}}^{I}$. Consequently, $\mathrm{x}^{\mathbf{g}^{+}-}$ $\mathbf{x}^{\mathbf{g}^{-}} \notin \mathcal{U}\left(A_{S(6)}\right)$. Yet $\mathbf{g}$ represents the homogeneous primitive partition identity

$$
1+3+7=2+4+5
$$

and thus $\mathbf{x}^{\mathbf{g}^{+}}-\mathbf{x}^{\mathbf{g}^{-}} \in \mathcal{G}\left(A_{S(6)}\right) \backslash \mathcal{U}\left(A_{S(6)}\right)$.

The other cases are proved in the same manner, so we will simply enumerate the vectors $\mathbf{g}^{+}, \mathbf{u}_{1}, \ldots, \mathbf{u}_{k}$ and coefficients $\lambda_{1}, \ldots, \lambda_{k}$ that are used in each case in Table 1 . Note that the matrices for $S(6)$ and $H(7)$ are identical. Also, for $S(5,4)$ and $H(4,3)$, we find that $\mathbf{g}^{+}=\frac{1}{2} \mathbf{u}_{1}+\frac{1}{2} \mathbf{u}_{2}$, so the argument simply shows that $g^{+}$ is not a vertex of its fiber.

Our computational method also yields the following, which we will need in order to complete the proof of Theorem 2.2. The exponential notation here indicates repetition.

Lemma 4.2. The Graver basis and the universal Gröbner basis coincide for the defining matrices of $S\left(3^{5}\right), H\left(3^{5}\right)$, $S\left(5,3,1^{7}\right), S\left(5,2^{8}\right), S\left(4,4,1^{5}\right)$, and $H\left(5,2^{12}\right)$. 


\begin{tabular}{|c|c|c|c|c|c|}
\hline Case & $\mathrm{g}^{+}$ & $\mathrm{g}^{-}$ & $k$ & $\mathbf{u}_{1}, \ldots, \mathbf{u}_{k}$ & $\lambda_{1}, \ldots, \lambda_{k}$ \\
\hline \multirow{3}{*}{$S(6)=H(7)$} & \multirow{3}{*}{$e_{1}+e_{3}+e_{7}$} & \multirow{3}{*}{$e_{2}+e_{4}+e_{5}$} & \multirow{3}{*}{3} & $e_{1}+2 e_{5}$ & 1 \\
\hline & & & & $2 e_{2}+e_{7}$ & 1 \\
\hline & & & & $e_{3}+2 e_{4}$ & 1 \\
\hline \multirow{2}{*}{$S(5,4)$} & \multirow{2}{*}{$e_{1,1}+e_{1,5}+e_{2,1}+e_{2,5}$} & \multirow{2}{*}{$e_{1,2}+e_{1,6}+2 e_{2,2}$} & \multirow{2}{*}{2} & $2 e_{1,5}+2 e_{2,1}$ & $1 / 2$ \\
\hline & & & & $2 e_{1,1}+2 e_{2,5}$ & $1 / 2$ \\
\hline \multirow{4}{*}{$S(4,3,2)$} & \multirow{4}{*}{$e_{5,1}+e_{1,2}+e_{3,2}+e_{1,3}$} & \multirow{4}{*}{$e_{1,1}+e_{2,2}+e_{4,2}+e_{3,3}$} & \multirow{4}{*}{4} & $e_{1,5}+e_{2,2}+2 e_{3,1}$ & $1 / 2$ \\
\hline & & & & $e_{1,1}+2 e_{2,4}+e_{3,1}$ & $1 / 2$ \\
\hline & & & & $e_{1,5}+2 e_{2,1}+e_{3,3}$ & $1 / 2$ \\
\hline & & & & $e_{1,1}+2 e_{2,3}+e_{3,3}$ & $1 / 2$ \\
\hline \multirow{3}{*}{$H(6,2)$} & \multirow{3}{*}{$e_{1,2}+e_{1,6}+2 e_{2,1}$} & \multirow{3}{*}{$e_{1,1}+e_{1,3}+e_{1,4}+e_{2,2}$} & \multirow{3}{*}{3} & $2 e_{1,4}+2 e_{1,5}$ & $1 / 2$ \\
\hline & & & & $2 e_{1,1}+e_{1,6}+e_{2,2}$ & $1 / 2$ \\
\hline & & & & $e_{1,2}+2 e_{1,3}+e_{1,6}$ & $1 / 2$ \\
\hline \multirow{2}{*}{$H(4,3)$} & \multirow{2}{*}{$e_{1,1}+2 e_{1,2}+e_{1,3}+e_{2,3}$} & \multirow{2}{*}{$2 e_{1,4}+3 e_{2,1}$} & \multirow{2}{*}{2} & $2 e_{1,1}+2 e_{1,3}+e_{2,3}$ & $1 / 2$ \\
\hline & & & & $4 e_{1,2}+e_{2,3}$ & $1 / 2$ \\
\hline
\end{tabular}

TABLE 1. Cases of the proof of Lemma 4.1.

\section{GRAVER COMPLEXITY AND THE REMAINDER OF THE PROOF}

Given integer matrices $C$ and $D$ of the same width and a number $c$, define the $c$-fold matrix

$$
M_{c}(C, D):=\underbrace{\left(\begin{array}{cccc}
D & D & \cdots & D \\
C & 0 & & 0 \\
0 & C & & 0 \\
& & \ddots & \\
0 & 0 & & C
\end{array}\right)}_{c \text { copies of } D} .
$$

Such matrices represent higher Lawrence liftings. Their special structure implies that as $c$ grows, the Graver bases of $M_{c}(C, D)$ eventually stabilize (see [Hoşten and Sullivant 07]), in the sense that the type $\left|i: \mathbf{u}^{(i)} \neq \mathbf{0}\right|$ of a vector $\mathbf{u}=\left(\mathbf{u}^{(1)}, \ldots, \mathbf{u}^{(c)}\right) \in$ $\mathcal{G}\left(M_{c}(C, D)\right)$ is bounded by a constant $g(C, D)$, the so-called Graver complexity of $C$ and $D$. In general, $g(C, D) \neq g(D, C)$. However, Graver complexity can be computed as follows.

Proposition 5.1. [Hoşten and Sullivant 07, Theorem 3.5] For any integer matrices $C$ and $D$ of the same width,

$$
g(C, D)=\max \left\{\|\mathbf{v}\|_{1}: \mathbf{v} \in \mathcal{G}(D \cdot \mathcal{G}(C))\right\}
$$

where the set $\mathcal{G}(D \cdot \mathcal{G}(C))$ denotes the Graver basis of the matrix obtained by multiplying each element of the Graver basis $\mathcal{G}(C)$ by the matrix $D$.

Lemma 5.2. For any fixed width $m, C=(1,1, \ldots, 1)$, and $D=(1,2, \ldots, m)$, we have $g(C, D)=2 m-3$ and $g(D, C) \leq 4 m-7$.
Proof. The first equality follows because the Graver basis of the $1 \times m$ matrix $C=(1,1, \ldots, 1)$ consists of all vectors $\mathbf{e}_{i}-\mathbf{e}_{j}, 1 \leq i \neq j \leq m$. Multiplying these elements by the $1 \times m$ matrix $D=(1,2,3, \ldots, m)$, we conclude by Proposition 5.1 that $g(C, D)$ equals the maximum 1-norm among the Graver basis elements of the matrix $(1,2,3, \ldots, m-1)$, which is known to be $2(m-1)-1=$ $2 m-3$ [Diaconis 96].

Similarly, we can compute $g(D, C)$. The Graver basis elements of $D=(1,2,3, \ldots, m)$ have a maximum 1 -norm of $2 m-1$. However, since no element in $\mathcal{G}(D)$ has only nonnegative entries, we have that $|C \mathbf{g}| \leq 2 m-3$. Hence by Proposition 5.1, $g(D, C)$ is bounded from above by the maximum 1-norm among the Graver basis elements of the matrix $(1,2,3, \ldots, 2 m-3)$, which is $2(2 m-3)-1=$ $4 m-7$.

We now apply this technique to our matrices. Let $S_{c, m-1}:=S\left((m-1)^{c}\right)$ and $H_{c, m}:=S\left(m^{c}\right)$. Then we have $A_{c, m-1}:=A_{S\left((m-1)^{c}\right)}=M_{c}(C, D)$ and $B_{c, m}:=$ $A_{H\left(m^{c}\right)}=M_{c}(D, C)$, where $C$ and $D$ are as above.

Lemma 5.3. The universal Gröbner basis equals the Graver basis for $A_{c, 3}$ and for $B_{c, 3}$ for every $c \in \mathbb{Z}_{>0}$.

Proof. For $c \leq 5$, this holds by Lemma 4.2 and Corollary 3.4. Suppose $\mathbf{g} \in \mathcal{G}\left(A_{c, 3}\right)$ for some $c>5$. By Lemma 5.2, the type of any Graver basis element of $A_{c, 3}$ is at most $2 \cdot 4-3=5$, so $\mathrm{g}$ represents a colorhomogeneous primitive partition identity with at most five colors. Define $\mathbf{g}^{\prime}$ by restricting $\mathbf{g}$ to the 20 coordinates that represent these five colors (eliminating only 
zeros). Then $\mathbf{g}^{\prime} \in \mathcal{G}\left(A_{5,3}\right)=\mathcal{U}\left(A_{5,3}\right)$. Thus, by applying Corollary 3.2 , we see that $\mathrm{g} \in \mathcal{U}\left(A_{c, 3}\right)$.

Similar arguments apply to $B_{c, 3}$. Here the type of any Graver basis element is bounded by $4 \cdot 3-7=5$, and the result follows from the equality of the universal Gröbner basis and Graver basis for $B_{5,3}$.

Lemma 5.4. The Graver basis and the universal Gröbner basis coincide for the defining matrices of $S(5)$, $S\left(5,3,1^{k}\right), S\left(5,2^{k}\right), S\left(4,4,1^{k}\right), H(6)$, and $H\left(5,2^{k}\right)$ for all natural numbers $k$.

Proof. We consider just the last case of $H\left(5,2^{k}\right)$, the others being analogous. If $k \leq 12$, the result follows from Lemma 4.2 and Corollary 3.4. So suppose $k>12$ and let $\mathbf{g} \in \mathcal{G}\left(A_{H\left(5,2^{k}\right)}\right)$. First observe that $A_{H\left(5,2^{k}\right)}$ can be obtained from $A_{H\left(5^{k}\right)}$ by deleting certain columns and zero rows thereafter. Thus, $\mathrm{g}$ can be lifted (by adding only components 0$)$ to $\mathbf{g}^{\prime} \in \mathcal{G}\left(A_{H\left(5^{k}\right)}\right)$ by Corollary 3.2. The type of any Graver basis element of $A_{H\left(5^{k}\right)}$ is bounded by $4 \cdot 5-7=13$. This implies that the type of $\mathrm{g}^{\prime}$ is at most 13. Therefore, it can be projected (by removing only zero components) to a Graver basis element $\mathbf{g}^{\prime \prime} \in \mathcal{G}\left(A_{H\left(5,2^{12}\right)}\right)$. By Lemma 4.2 , we conclude that $\mathbf{g}^{\prime \prime} \in$ $\mathcal{U}\left(A_{H\left(5,2^{12}\right)}\right.$. Applying Corollary 3.2 twice, we conclude that $\mathbf{g} \in \mathcal{U}\left(A_{H\left(5,2^{k}\right)}\right)$ and thus that $\mathcal{U}\left(A_{H}\right)=\mathcal{G}\left(A_{H}\right)$ as claimed.

Remainder of proof of Theorem 2.2. We can now complete the proof of Theorem 2.2. The "only if" direction follows immediately from Lemma 4.1 and Corollary 3.4. The "if" direction will follow from straightforward case analysis. Suppose that $S$ is a scroll that does not dominate $S(6), S(5,4)$, or $S(4,3,2)$. Then one of the following holds:

Case 1: $n_{1} \leq 4$. Then $S \preceq S_{c, 3}$ for some $c$ and thus equality holds by Lemma 5.3 .

Case 2: $n_{1}=n_{2}=5$. Then $n_{3} \leq 2$ to avoid dominating $S(4,3,2)$. Thus, we have $S=S(4,4,1, \ldots, 1)$.

Case $3: n_{1}=5$ or $6, n_{2} \leq 3$. Then $S \preceq S(5,2, \ldots, 2)$.

Case 4: $n_{1}=5$ or $6, n_{2}=4$. Then $n_{3} \leq 2$ to avoid dominating $S(4,3,2)$. Thus, we have $S \preceq S(5,3,1, \ldots, 1)$.

By Corollary 3.4 and Lemma 5.4, the universal Gröbner basis and Graver basis coincide for the scrolls $S$ in Cases 2, 3, and 4 .
Similarly, suppose $H$ does not dominate $H(7), H(6,2)$, or $H(4,3)$. Then one of the following holds:

Case 1: $n_{1} \leq 3$. Then $H \preceq H_{c, 3}$ for some $c$ and thus equality holds by Lemma 5.3.

Case 2: $n_{1}=4$ or 5 . Then $n_{2} \leq 2$ to avoid dominating $H(4,3)$. Then $H \preceq H(5,2, \ldots, 2)$.

Case 3: $n_{1}=6$. Then $n_{2} \leq 1$ to avoid dominating $H(6,2)$. But then $H$ has the same toric ideal as $H(6)$.

By Corollary 3.4 and Lemma 5.4, the universal Gröbner basis and Graver basis coincide for $H$ in Cases 2 and 3 .

Although equality of Graver and Gröbner bases implies that the Graver and Gröbner complexities are equal, the converse does not hold. We close with a negative answer to the question of Hemmecke and Nairn: $N$-fold matrices with fixed Gröbner and Graver complexities do not preserve equality of universal Gröbner and Graver bases. Specifically, we have the following.

Corollary 5.5. There exist integer matrices $A$ and $B$, of appropriate dimensions, satisfying $\mathcal{G}(A)=\mathcal{U}(A)$, and hence $u(A, B)=g(A, B)$, but that still satisfy $\mathcal{U}\left(M_{N}(A, B)\right) \subsetneq \mathcal{G}\left(M_{N}(A, B)\right)$ for all $N$-folds for $N>1$.

Proof. One such example is given by the rational normal curve $S(5)$ in $\mathbb{P}^{5}$. The corresponding matrices are $A=$ $(1,1,1,1,1,1)$ and $B=(1,2,3,4,5,6)$. By Lemma 5.4 , the Graver and universal Gröbner bases of the curve coincide. The Graver and Gröbner complexities thus agree by [Hemmecke and Nairn 09, Theorem 1]. On the other hand, the matrices $M_{N}(A, B)$, by definition, correspond to $N$-dimensional scrolls $S(5,5, \ldots, 5)$. But by Theorem 2.2, these dominate the minimal counterexamples, and therefore the Graver and universal Gröbner bases of these $N$-fold matrices do not agree.

\section{REFERENCES}

[Conca et al. 07] A. Conca, E. De Negri, and M. E. Rossi. "Contracted Ideals and the Gröbner Fan of the Rational Normal Curve." Algebra Number Theory 1:3 (2007), 239268 .

[Diaconis 96] P. Diaconis, R. Graham, and B. Sturmfels. "Primitive Partition Identities." In Combinatorics: Paul Erdős is Eighty, edited by D. Miklós, V. T. Sós, and 
T. Szonyi, pp. 173-192. János Bolyai Mathematical Society, Budapest, 1996.

[Drton et al. 09] M. Drton, B. Sturmfels and S. Sullivant. Lectures on Algebraic Statistics, Oberwolfach Seminars 39. Birkhäuser, 2009.

[Eisenbud and Harris 87] D. Eisenbud and J. Harris. "On Varieties of Minimal Degree (a Centennial Account)." In Algebraic Geometry, Bowdoin, 1985, Proceedings of Symposia in Pure Mathematics 46:1, pp. 3-13, 1987.

[Fulton 93] W. Fulton. Introduction to Toric Varieties, Ann. of Math. Stud. 131. Princeton University Press, 1993.

[Hemmecke and Nairn 09] R. Hemmecke and K. Nairn. "On the Gröbner Complexity of Matrices." Journal of Pure and Applied Algebra 213 (2009), 1558-1563.

[Hoşten and Sullivant 07] S. Hoşten and S. Sullivant. "Finiteness Theorems for Markov Bases of Hierarchical Models." Journal of Combinatorial Theory, Series A 114:2 (2007), 311-321.
[Petrović 08] S. Petrović. "On the Universal Gröbner Bases of Varieties of Minimal Degree." Math. Res. Lett. 15:6 (2008), 1211-1221.

[Santos and Sturmfels 03] F. Santos and B. Sturmfels. "Higher Lawrence Configurations." J. Combin. Theory Ser. A 103 (2003), 151-164.

[Sturmfels 92] B. Sturmfels. "Asymptotic Analysis of Toric Ideals." Memoirs of the Faculty of Science, Kyushu University, Ser. A 46 (1992), 217-228.

[Sturmfels 96] B. Sturmfels. Gröbner Bases and Convex Polytopes, University Lecture Series 8. American Mathematical Society, 1996.

[Sturmfels and Thomas 97] B. Sturmfels and R. R. Thomas. "Variation of Cost Functions in Integer Programming." Mathematical Programming 77 (1997), 357-387.

[Villarreal 01] R. Villarreal. Monomial Algebras, Monographs and Textbooks in Pure and Applied Mathematics 238. Marcel Dekker, 2001.

Tristram Bogart, Department of Mathematics, Los Anders University, Bogotá, Colombia (tc.bogart22@uniandes.edu.co)

Ray Hemmecke, Zentrum Mathematik, M9, Technische Universität München, D-85747 Garching bei München, Germany (hemmecke@ma.tum.de)

Sonja Petrović, Department of Statistics, Pennsylvania State University, 326 Thomas Building, University Park, PA 16802 (petrovic@psu.edu) 